\title{
Antiepileptic drug-induced severe cutaneous adverse reactions and $H L A$ alleles: A report of five cases with lymphocyte activation test
}

\author{
Eun-Young Kim ${ }^{1,2}(\mathbb{D})$ Mi-Yeong Kim $^{3}(\mathbb{D})$, Chan Sun Park ${ }^{4}\left(\mathbb{D}\right.$, Jae-Hyeog Choi ${ }^{5}$ (D) Jong-Lyul Ghim ${ }^{1,2}(\mathbb{D})$, \\ Ho-Sook Kim ${ }^{2,6}(\mathbb{D})$ and Jae-Gook Shin ${ }^{1,2,6 *}$ (D) \\ ${ }^{1}$ Department of Clinical Pharmacology, Inje University College of Medicine, Busan Paik Hospital, Busan 47392, Republic of Korea \\ ${ }^{2}$ Center for Personalized Precision Medicine of Tuberculosis, Inje University College of Medicine, Busan 47392, Republic of Korea \\ ${ }^{3}$ Department of Internal Medicine, Inje University College of Medicine, Busan Paik Hospital, Busan 47392, Republic of Korea \\ ${ }^{4}$ Department of Internal Medicine, Inje University College of Medicine, Haeundae Paik Hospital, Busan 48108, Republic of Korea \\ ${ }^{5}$ Department of Microbiology and Immunology, Inje University College of Medicine, Busan 47392, Republic of Korea \\ ${ }^{6}$ Department of Pharmacology and PharmacoGenomics Research Center, Inje University College of Medicine, Busan 47392, Republic of Korea \\ *Correspondence: J. G. Shin; Tel: +82-51-890-6709, Fax: +82-51-893-1232, E-mail: phshinjg@gmail.com
}

Check for updates

Received 3 Jun 2019

Revised 21 Jun 2019

Accepted 21 Jun 2019

\section{Keywords}

Antiepileptic drugs, HLA class I alleles, Lymphocyte activation test, Severe cutaneous adverse reactions

pISSN: 2289-0882 eISSN: $2383-5427$
Antiepileptic drugs (AEDs) can induce severe cutaneous adverse reactions (SCARs) such as Stevens-Johnson syndrome (SJS), toxic epidermal necrolysis (TEN), and drug reaction with eosinophilia and systemic symptoms (DRESS) syndrome. We performed HLA genotyping and lymphocyte activation tests (LATs) for five AED-induced SCAR patients (three males and two females; aged 40-66 years old). Three patients were treated with carbamazepine (CBZ) for pain control, one was treated with phenytoin (PHT) for seizure prevention, and one was treated with valproic acid (VPA) for seizure prevention. One patient was diagnosed with CBZ-induced DRESS syndrome and the remaining patients were diagnosed with SJS. All patients recovered from SCARs after stopping suspicious drugs and supportive care. LATs were conducted to confirm the culprit drug responsible for inducing SCARs; and LAT results were positive for the suspected culprit drugs, in all except in one case. $H L A-A,-B$, and $-C$ alleles were determined using PCR-sequence-based typing method. The common alleles of $H L A$ were $-A^{*} 02: 01,-B^{*} 51: 01$, and $-C^{*} 03: 04$ which were carried by three patients $(60 \%)$ for each allele. The patient with CBZ-induced DRESS syndrome carried the HLA$A *$ 31:01 allele. One patient with CBZ-induced SJS and one patient with VPA-induced SJS carried the $H L A-B^{*} 15: 11$ allele. No patients carried the $H L A-B^{*}$ 15:02 allele, which is a known risk allele of AED-induced SCARs. Further investigation of the three common alleles found in the five AED-induced SCARs patients is needed. We demonstrated the usefulness of LAT for confirming the culprit drug.
Antiepileptic drugs (AEDs) can induce life-threatening severe cutaneous adverse reactions (SCARs) such as Stevens-Johnson syndrome (SJS), toxic epidermal necrolysis (TEN), and drug

Copyright (C) 2019 Translational and Clinical Pharmacology (a) It is identical to the Creative Commons Attribution Non-Commercial License (http://creativecommons.org/licenses/by-nc/3.0/).

@ This paper meets the requirement of KS X ISO 9706, ISO 9706-1994 and ANSI/NISO Z.39.48-1992 (Permanence of Paper).

Reviewer

This article reviewed by peer experts who are not TCP editors. reaction with eosinophilia and systemic symptoms (DRESS) syndrome.[1,2] Drug reactions are usually caused by immunemediated mechanisms, and the associations between culprit drugs and HLA alleles have been studied.[3] The relationship between AED-induced SCARs and HLA alleles has been demonstrated for carbamazepine (CBZ) with $H L A-B * 15: 02$ among Han Chinese.[4] CBZ-induced cutaneous adverse drug reactions, including drug induced hypersensitivity syndrome, SJS/TEN, and others, were associated with $H L A-A * 31: 01$ in Japanese[5] and CBZ-induced DRESS syndrome was strongly 
associated with $H L A-A * 31: 01$ in Spanish.[6] $H L A-B * 15: 13$ and $H L A-B^{*}$ 15:02 were associated with phenytoin (PHT)-induced SCARs in Malays.[7] The cross-reactivity between aromatic AEDs, CBZ, PHT, phenobarbital, and lamotrigine (LTG), is well established.[2] SCARs caused by non-aromatic AEDs, such as valproic acid (VPA) and levetiracetam, are less common than those caused by aromatic AEDs.[1,2] In particular, VPA hypersensitivity has often been reported in patients simultaneously taking other medications, especially LTG.[8] We report five cases of AED-induced SCARs including clinical findings, HLA class I genotypes, and lymphocyte activation test (LAT) results.

Written informed consent was obtained from all five patients according to the protocol approved by the Institutional Review Board of Inje University Busan Paik Hospital, Busan, South Korea (IRB No. 05-059). Clinical data collection from electronic medical records, HLA class I genotyping, and LAT were conducted for all five patients.

\section{HLA genotyping}

Peripheral blood samples were collected from all five patients and genomic DNA was extracted from peripheral blood mononuclear cells (PBMCs) using the QIAamp Blood Mini Kit (QIAGEN, Hilden, Germany). HLA-A, -B, and -C genotypes were identified using the PCR-sequence-based typing (SBT) method (SBT Engine software version 2.20, GenDx, Utrecht, Netherland).

\section{Lymphocyte activation test (LAT)}

Using the PBMCs, LATs[9] were performed to test for hypersensitivity reaction to the following AEDs: CBZ, VPA, LTG, phenobarbital, and PHT, using published methods.[10] Tests were performed in the presence of $0.5 \mathrm{ug} / \mathrm{mL}$ phytohemagglutinin A (PHA, Sigma-Aldrich, St. Louis, MO, USA) for a positive control or in the absence of the reagent for negative control. Stimulation index $(\mathrm{SI})>2.0$ was interpreted as a positive result.

\section{Case Report}

\section{Clinical characteristics}

Demographic, clinical information, and causality assessments of the five patients are summarized in Table 1. Causality assessments were performed using the World Health Organization (WHO)-Uppsala Monitoring Centre (UMC) system.[11]

\section{Case 1}

A 59-year-old female presented to the dermatology outpatient department (OPD) with erythematous patches across her whole body, without Nikolsky's sign. Symptom onset was one week prior to presentation. Oral mucosal erosion and conjunctival injection were observed. The patient reported itching without pain. For the past ten years, the patient had been taking hormone replacement therapy (tibolone) for postmenopausal symptoms and an antihypertensive drug (lercanidipine). Two weeks prior to symptom onset, the patient began taking the following new drugs twice daily for back pain: CBZ (100 mg), tramadol (37.5 mg) and acetaminophen (325 mg) in combination, aceclofenac (100 mg), cimetidine (200 mg), and piprinhydrinate (3 mg). On the day of presentation, routine hematological test (complete blood cell count, $\mathrm{CBC}$ ), liver function, renal function, and radiology exam (simple chest $\mathrm{X}$-ray) were normal. The

Table 1. Demographic information, clinical findings, and causality assessments in the AED-induced SCARs patients

\begin{tabular}{|c|c|c|c|c|c|c|c|}
\hline Case & Sex/age & Diagnosis & $\begin{array}{l}\text { WHO-UMC } \\
\text { criteri }^{\mathrm{a}}\end{array}$ & $\begin{array}{l}\text { Culprit } \\
\text { drug }\end{array}$ & Manifestation & $\begin{array}{l}\text { Concomitant } \\
\text { medications }\end{array}$ & Co-morbidities \\
\hline 1 & $\mathrm{~F} / 59$ & SJS & Probable & $\mathrm{CBZ}$ & $\begin{array}{l}\text { Maculopapular eruption across the } \\
\text { whole body; mucosal involvement of } \\
\text { the eye and oral cavity }\end{array}$ & $\begin{array}{l}\text { Tramadol, } \\
\text { acetaminophen, } \\
\text { aceclofenac, cimetidine, } \\
\text { piprinhydrinate }\end{array}$ & $\begin{array}{l}\text { Postmenopausal } \\
\text { syndrome, } \\
\text { hypertension, } \\
\text { back pain }\end{array}$ \\
\hline 2 & $\mathrm{M} / 62$ & SJS & Probable & PHT & $\begin{array}{l}\text { Erythematous patches across the } \\
\text { whole body; mucosal involvement of } \\
\text { the eye and oral cavity; eosinophilia, } \\
\text { elevation of hepatic enzymes }\end{array}$ & $\begin{array}{l}\text { Meropenem, } \\
\text { aceclofenac }\end{array}$ & $\begin{array}{l}\text { Bacterial meningitis, } \\
\text { cerebral infarction }\end{array}$ \\
\hline 3 & $\mathrm{~F} / 47$ & SJS & Probable & $\mathrm{CBZ}$ & $\begin{array}{l}\text { Erythematous patches across the } \\
\text { whole body; mucosal involvement of } \\
\text { the eye and oral cavity }\end{array}$ & None & $\begin{array}{l}\text { Carpal tunnel } \\
\text { syndrome }\end{array}$ \\
\hline 4 & $\mathrm{M} / 40$ & SJS & Probable & VPA & $\begin{array}{l}\text { Erythematous patches across the } \\
\text { whole body; mucosal involvement of } \\
\text { the oral cavity }\end{array}$ & $\begin{array}{l}\text { Gabapentin, } \\
\text { alprazolam, oxiracetam }\end{array}$ & $\begin{array}{l}\text { Epileptic disorder, } \\
\text { fatty liver }\end{array}$ \\
\hline 5 & $\mathrm{M} / 66$ & DRESS & Probable & $\mathrm{CBZ}$ & $\begin{array}{l}\text { Purpura on lower extremities; fever; } \\
\text { neutropenia; eosinophilia; elevation } \\
\text { of hepatic enzymes; lung infiltration, } \\
\text { lymph node enlargement }\end{array}$ & Thioctacid & $\begin{array}{l}\text { Unstable angina, } \\
\text { neuropathic pain }\end{array}$ \\
\hline
\end{tabular}

AED, anti-epileptic drug; SCARs, severe cutaneous adverse reactions; CBZ, carbamazepine; PHT, phenytoin; VPA, valproic acid; SJS, StevensJohnson syndrome; DRESS, drug reaction with eosinophilia and systemic symptoms. ${ }^{a}$ Causality assessment followed the World Health Organization-Uppsala Monitoring Centre (UMC) system.[11] 
patient was afebrile, and an electrocardiogram (ECG) showed normal sinus rhythm. Ophthalmologic examination revealed moderate follicular injection and discharge in both conjunctivas. Excision skin biopsy (from the leg) confirmed erythema multiforme (EM). The patient was diagnosed with SJS with mucosal involvement of the eye and oral cavity and CBZ was suspected to be the culprit drug. All newly started drugs were stopped. Systemic corticosteroid, oral methylprednisolone (24 $\mathrm{mg} /$ day) and topical corticosteroid, desonide (500 ug/g) were prescribed. Oral antihistamine was prescribed for itching, and fluorometholone eye drops were also prescribed. Oral corticosteroid was tapered off after one week. The patient recovered without complication.

\section{Case 2}

A 62-year-old male hospitalized with bacterial meningitis and cerebral infarction was referred to the dermatology department with erythematous patches across his whole body. Symptom onset was one week prior to dermatology consultation. Oral mucosal erosion and conjunctival injection were observed, as well as some blistering on the body. Forty-five days prior to dermatologic symptom onset, meropenem was started as an antiinfective drug, and PHT was commenced as an AED. The patient was also intermittently administered aceclofenac for pain relief. Laboratory tests revealed moderate eosinophilia and mild elevation of hepatic enzymes (WBC-Hb-Plt $14.47 \times 10^{9} / \mathrm{L}-12.8$ g/dL- $419 \times 10^{9} / \mathrm{L}$, neutrophil $77 \%$, lymphocyte $8 \%$, monocyte $5 \%$, eosinophil 7\%, basophil 2\%, and AST/ALT/ALP 38/62/484 U/L). Renal function was within the normal range (BUN/Cr 20/0.37 $\mathrm{mg} / \mathrm{dL}$ ). Excision skin biopsy (from the leg) confirmed EM. The patient was diagnosed with SJS with mucosal involvement of the eye and oral cavity. While PHT was suspected to be the culprit drug, meropenem could not be excluded as the possible culprit drug. Thus, PHT and meropenem were changed to ceftriaxone and levetiracetam. Tramadol was used to treat pain instead of non-steroidal anti-inflammatory drugs. Cyclosporine $(125 \mathrm{mg}$, twice daily), desonide (500 ug/g), and fluorometholone eye drops were prescribed. After two weeks' treatment, symptoms and laboratory abnormalities improved, and cyclosporine was stopped. The patient recovered without complication.

\section{Case 3}

A 47-year old female presented to the emergency department (ED) with erythematous patches across her whole body. Symptom onset was one week prior to presentation. Oral mucosal erosion and conjunctiva injection were observed. The patient had taken CBZ $200 \mathrm{mg}$ and acetaminophen (unknown dose) for carpal tunnel syndrome for two weeks prior to symptom onset and stopped taking the drugs one week ago. On the day of presentation, routine hematological test $(\mathrm{CBC})$, liver function, renal function, and radiology exam (simple chest $\mathrm{X}$-ray) were normal, and ECG showed normal sinus rhythm. Excision skin biopsy (from the leg) confirmed EM. The patient was diagnosed with SJS with mucosal involvement of the eye and oral cavity, and CBZ was suspected to be the culprit drug. Oral methylprednisolone (12 mg, twice a day), and desonide (500 ug/g) were prescribed. Oral antihistamine was prescribed for itching, and fluorometholone eye drops were also prescribed. Oral corticosteroids were tapered off after two weeks. The patient recovered without complications.

\section{Case 4}

A 40-year old male presented to the ED with erythematous patches across his whole body. Symptom onset was four days prior to presentation. Oral mucosal erosion was observed. The patient took VPA $500 \mathrm{mg}$ due to seizures following traffic accident induced brain trauma, but the dosage and duration of taking the VPA were not accurate. Eight months prior to the current presentation, levetiracetam was prescribed at our hospital, before the patient was transferred to another hospital for supportive care. The time of change from levetiracetam to VPA could not be verified. The prescription medication record confirmed that VPA was administered at least two weeks before symptom onset. On the day of presentation, laboratory tests showed leukopenia, thrombocytopenia, and elevation of total bilirubin and hepatic enzymes (WBC-Hb-Plt 3.02×10 $/ \mathrm{L}-15.5$ g/dL- $96 \times 10^{9} / \mathrm{L}$, neutrophil $62.2 \%$, lymphocyte $20.9 \%$, monocyte $16.6 \%$, eosinophil $0.0 \%$, basophil $0.3 \%$, total protein/albumin 4.7/2.6 g/dL, AST/ALT/ALP 211/438/662 U/L, total bilirubin $11.1 \mathrm{mg} / \mathrm{dL}$, glucose $179 \mathrm{mg} / \mathrm{mL}$, and BUN/Cr 10/0.44 mg/dL). Simple chest X-ray showed subsegmental atelectasis in the right lower lung field; abdominal CT showed fatty liver without other hepatic abnormality, and ECG showed sinus tachycardia. The patient was diagnosed with SJS with mucosal involvement of the oral cavity and VPA was suspected to be the culprit drug. Intravenous methylprednisolone ( $60 \mathrm{mg} /$ day), and desonide (500 ug/ g) were prescribed. Oral antihistamine was prescribed for itching. Corticosteroids were tapered off after two weeks. There was no further elevation of hepatic enzymes and total bilirubin.

\section{Case 5}

A 66-year old male presented to the OPD with fever due to infection, complaining of a "cold sensation" and purpura on the lower extremities. For the past year, the patent had been taking aspirin (100 mg), clopidogrel (75 mg/day), valsartan (80 $\mathrm{mg} /$ day), pravastatin (20 mg/day), nicorandil (5 mg/day), and trimetazidine (20 mg/day) for unstable angina. Three weeks prior to symptom onset, the patient began taking CBZ (200 mg) and thioctacid (200 mg) for tingling sensation in his hands. On the day of presentation, laboratory tests showed neutropenia, eosinophilia, and elevation of hepatic enzymes (WBC-Hb-Plt $2.37 \times 10^{9} / \mathrm{L}-12.3 \mathrm{~g} / \mathrm{dL}-198 \times 10^{9} / \mathrm{L}$, neutrophil $14 \%$, lymphocyte $36 \%$, monocyte $14 \%$, eosinophil $35 \%$, basophil $0 \%$, total protein/albumin 7.5/4.2 g/dL, AST/ALT/ALP 67/76/332 U/ $\mathrm{L}$, total bilirubin $0.7 \mathrm{mg} / \mathrm{dL}$, glucose $128 \mathrm{mg} / \mathrm{mL}$, and BUN/ Cr 12/0.9 mg/dL). HBsAg and HCV antibodies were negative. 
Table 2. HLA alleles and LAT results in the cases

\begin{tabular}{ccccc}
\hline Case & HLA-A & HLA-B & HLA-C & Positive for LAT \\
\hline 1 & $* 02: 01 / * 26: 01$ & $* 15: 11 / * 51: 01$ & $* 03: 04 / 15: 02$ & Negative $^{a}$ \\
2 & $* 24: 02 / * 26: 02$ & $* 51: 01 / * 51: 01$ & $* 14: 02 / * 14: 02$ & PHT $^{a}$ \\
3 & $* 02: 01 / * 02: 01$ & $* 35: 01 / * 51: 01$ & $* 03: 04 / * 14: 02$ & CBZ \\
4 & $* 02: 01 / * 02: 10$ & $* 15: 11 / * 40: 06$ & $* 03: 04 / 08: 01$ & VPA, PHT, LTG \\
5 & $* 24: 02 / * 31: 01$ & $* 07: 02 / * 58: 01$ & $* 03: 02 / * 07: 02$ & CBZ, VPA, PHT \\
\hline
\end{tabular}

HLA, human leukocyte antigen; LAT, lymphocyte activation test; PHT, phenytoin; CBZ, carbamazepine; VPA, valproic acid; LTG, lamotrigine. ${ }^{2}$ LAT was performed on the suspected causative agent only, not other anti-epileptic drugs.

CMV IgG antibody was positive but IgM antibody was negative. Titers of EBV VCA IgM/VCA IgG/EA IgG/EBNA IgG were 0.33/1.07/0.29/2.40 IU/mL. Peripheral blood cultures (two pairs of aerobe and non-aerobe bottles) were negative for bacterial growth. Bone marrow examination showed normocellular marrow with eosinophilic hyperplasia. Excision skin biopsy (from the leg) revealed spongiosis, extravasated red blood cells, and superficial perivascular lymphoplasmacytic infiltration. The patient was hospitalized. Lung infiltration was detected on the day of hospitalization. High-resolution chest CT showed groundglass opacity in the right upper lobe, bilateral pleural effusion, and enlarged lymph nodes at the neck, mediastinum, and both axillae. The patient was diagnosed with CBZ-induced DRESS syndrome, but not an infection. Intravenous methylprednisolone, $60 \mathrm{mg} /$ day, was started. Fever and eosinophilia did not improve over the following week. The dose of methylprednisolone was increased to $120 \mathrm{mg}$ /day. Then the symptoms and abnormal laboratory findings were gradually resolved. The patient experienced flareup twice during management. Corticosteroids were slowly tapered off over nine months.

\section{HLA alleles and LAT results}

$H L A-A,-B$, and $-C$ alleles and LAT results are shown in Table 2. The common $H L A$ alleles were $-A * 02: 01,-B * 51: 01$, and $-C^{*} 03: 04$; these were carried by $60 \%$ of patients in each allele. Cases 1 (CBZ-induced SJS) and 4 (VPA-induced SJS) carried HLA-B*15:11. Case 5 (CBZ-induced DRESS) carried HLA$A * 31: 01$ and $H L A-B * 58: 01$.

For Cases 1 and 2, LATs were performed on the suspected agent (CBZ and PHT, respectively), only. For Cases 3-5, LATs were performed for the following AEDs: CBZ, VPA, LTG, phenobarbital, and PHT (results are shown in Figure 1). The suspected culprit drugs were confirmed by LAT, except for Case 1 which was negative for CBZ. Case 4 LAT results were positive for PHT and LTG, as well as the suspected agent, VPA. Case 5 LAT results were positive for several agents including VPA and PHT as well as the suspected agents, CBZ. Case 5 showed positive possibility in LTG (lamictal ${ }^{\circledR}$ in Figure 1).

\section{Discussion}

The five cases reported here used aromatic AEDs CBZ and PHT, and nonaromatic AEDs VPA for prophylactic seizure

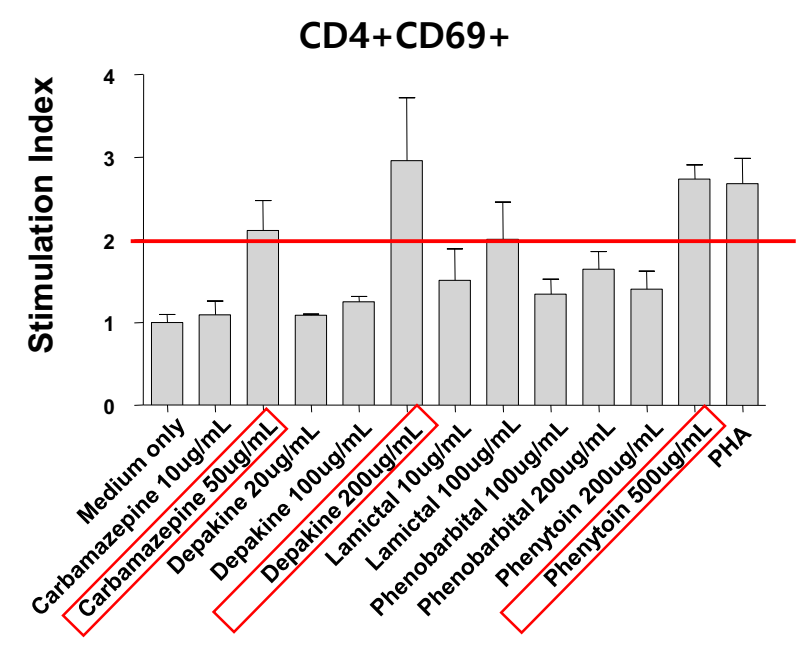

Figure 1. Lymphocyte activation test showing carbamazepine, valproic acid (depakine), and phenytoin positive (stimulation index $>2$ ) by CD69 upregulation of flow cytometric analysis. CD69 upregulation on CD4 $\mathrm{T}$ cells from Case 5 at $72 \mathrm{hr}$ after culture medium (negative control), different drugs, or PHA (phytohemagglutinin A, positive control) stimulation.

control/actual seizure control, or pain control. The latency to skin reactions after drug exposure was about two to five weeks post-drug administration. Case 4 had an unclear history of VPA dosage and duration. The diagnosis was SJS in four cases and DRESS syndrome in one case.

The Korean allele frequencies of the five cases' common alleles were $17.62 \%$ for $-A * 02: 01,9.54 \%$ for $-B^{*} 51: 01$, and $8.97 \%$ for $-C^{*} 03: 04$.[12] The frequencies of these alleles were higher among the five presented cases $(60 \%)$ than in the Korean population. Expression of $H L A-B^{*} 15: 11$ alleles were detected in two cases $(40.0 \%)$. Two cases had homozygous $H L A-B * 51: 01$ and $H L A-B * 15: 11 / * 51: 01$ alleles. $H L A-B * 51: 01$ carriers were all aromatic AED-induced SCAR patients (Cases 1-3). In a published study, three of four LTG-induced SCARs patients also carried $-B * 51: 01$.[13] Other drug metabolic enzyme-related genotypes, such as CYP2C9, were not investigated in the presented five cases. Further investigation would be helpful to understand the reaction mechanisms. There was no $H L A-B * 15: 02$ carriers among the five cases studied. This allele is a known risk allele in 
aromatic-structured AEDs-induced SCARs.[4,7] Only one of the three CBZ-induced SCARs cases carried $H L A-A * 31: 01$, also an identified risk allele of CBZ-induced cutaneous adverse drug reactions. [5]

The reported sensitivity and specificity of LAT were $16.75 \%$ and $97.8 \%$, respectively, as an in vitro diagnostic method used to detect drug sensitization at the cellular level for nonimmediate $\mathrm{T}$ cell-mediated drug hypersensitivity reactions. [9] The high specificity suggests that LAT can be used to identify a causative agent without re-challenging the drug in the patient. Generally, the sensitivity of the LAT has been reported as low, but our results confirmed the clinically suspicious drug to be the causative agent for SCARs in all except in one case (Case 1). Two patients (Cases 4 and 5) showed positive results in unused aromatic AEDs in PHT/LTG and CBZ/PHT, respectively. This could be thought of as a cross-reaction between AEDs. The symptoms may have presented after using positive-result drugs. Thus, the LAT test may be a useful tool for predicting and preventing adverse reactions.

VPA-induced SCARs are rare, and usually occur after concomitant use with aromatic AEDs, such as LTG. $[1,2,8]$ Crossreactivity among aromatic AEDs has been explained by the 'hapten hypothesis' or the 'pharmacological interaction theory'. $[2,14]$ These suggest that the difference in structure between aromatic and nonaromatic AEDs may affect interactions with HLA. Interestingly, before the use of VPA, Case 4 used a nonaromatic AED (levetiracetam). However, this patient was diagnosed with SCARs, DRESS syndrome.

We introduced five cases of AED-induced SCARs (SJS and DRESS syndrome) with HLA class I alleles and confirmed causative agents using LAT. The suspected culprit drugs were CBZ, PHT, and VPA, and each was confirmed to be the causative agent by LAT, except in one case. The common HLA alleles among the cases $(60 \%)$ were $-A^{*} 02: 01,-B^{*} 51: 01$, and $-C^{*} 03: 04$. The results suggest that Korean individuals with the alleles may be susceptible to AED-induced SCARs. Further investigations are necessary to confirm these findings. Two cases that both used CBZ carried HLA-A*31:01 (in DRESS syndrome) and $-B^{*} 15: 11$ (in SJS), respectively. Both LAT and HLA genotyping may be helpful to prevent SCARs due to AEDs.

\section{Acknowledgments}

This work was supported by the National Research Foundation of Korea (NRF) grant funded by the Korea government (MSIT) (No. 2018R1A5A2021242). The authors extend their gratitude to Eun-Young Cha for HLA genotyping.

\section{Conflict of interest}

- Authors: The authors declare no conflicts of interest exist regarding this study.

- Reviewers: Nothing to declare

- Editors: Nothing to declare

\section{References}

1. Knowles SR, Shapiro LE, Shear NH. Anticonvulsant hypersensitivity syndrome: Incidence, prevention and management. Drug Saf 1999;21:489501.

2. Blaszczyk B, Lasoń W, Czuczwar SJ. Antiepileptic drugs and adverse skin reactions: An update. Pharmacol Rep 2015;67:426-434. doi: 10.1016/j. pharep.2014.11.009.

3. Roujeau JC. Immune mechanisms in drug allergy. Allergol Int 2006;55:2733.

4. Chung WH, Hung SI, Hong HS, Hsih MS, Yang LC, Ho HC, et al. Medical genetics: A marker for Stevens-Johnson syndrome. Nature 2004;428:486.

5. Ozeki T, Mushiroda T, Yowang A, Takahashi A, Kubo M, Shirakata Y, et al. Genome-wide association study identifies HLA-A ${ }^{\star} 3101$ allele as a genetic risk factor for carbamazepine-induced cutaneous adverse drug reactions in Japanese population. Hum Mol Genet 2011;20:1034-1041. doi: 10.1093/hmg/ddq537.

6. Ramírez E, Bellón T, Tong HY, Borobia AM, de Abajo FJ, Lerma V, et al. Significant HLA class I type associations with aromatic antiepileptic drug (AED)-induced SJS/TEN are different from those found for the same AEDinduced DRESS in the Spanish population. Pharmacol Res 2017;115:168178. doi: 10.1016/j.phrs.2016.11.027.

7. Chang CC, Ng CC, Too CL, Choon SE, Lee CK, Chung WH, et al. Association of HLA-B*15:13 and HLA-B*15:02 with phenytoin-induced severe cutaneous adverse reactions in a Malay population. Pharmacogenomics J 2017;17:170-173. doi: 10.1038/tpj.2016.10.

8. Mansur AT, Pekcan Yaşar S, Göktay F. Anticonvulsant hypersensitivity syndrome: Clinical and laboratory features. Int J Dermatol 2008;47:11841189. doi: 10.1111/j.1365-4632.2008.03827.x.

9. Romano A, Torres MJ, Castells M, Sanz ML, Blanca M. Diagnosis and management of drug hypersensitivity reactions. J Allergy Clin Immunol 2011;127:S67-S73. doi: 10.1016/j.jaci.2010.11.047.

10. Kim EY, Seol JE, Choi JH, Kim NY, Shin JK. Allopurinol-induced severe cutaneous adverse reactions: A report of three cases with the HLAB*58:01 allele who underwent lymphocyte activation test. Transl Clin Pharmacol 2017;25:63-66.

11. World Health Organization (WHO), Uppsala Monitoring Centre. The use of the WHO-UMC system for standardised case causality assessment. http://www.who.int/medicines/areas/quality_safety/safety_efficacy/WHOcausality_assessment.pdf Accessed 30 May 2019

12. In JW, Roh EY, Oh S, Shin S, Park KU, Song EY. Allele and haplotype frequencies of human leukocyte antigen-A, $-B,-C$, -DRB1, and -DQB1 from sequence-based DNA typing data in Koreans. Ann Lab Med 2015;35:429-435. doi: 10.3343/alm.2015.35.4.429.

13. Kim EY, Ji KH, Kim HJ, Jung HE, Cha EY, Shin J. HLA-A*24:02/B*51:01 haplotype and lamotrigine-induced cutaneous adverse drug reactions in Koreans. Transl Clin Pharmacol 2016;24:143-146.

14. Ghosh K, Banerjee G, Ghosal AK, Nandi J. Cutaneous drug hypersensitivity: Immunological and genetic perspective. Indian J Dermatol 2011;56: 137-144. doi: 10.4103/0019-5154.80402. 